\title{
13. DATA REPORT: NEOGENE DIATOMS RECOVERED ON LEG 150X ${ }^{1}$
}

\author{
Lloyd H. Burckle ${ }^{2}$
}

\section{INTRODUCTION AND METHODS}

This data report lists Neogene diatoms recovered from three drill sites along the New Jersey coastal plain (Atlantic City, Cape May, and Island Beach; Tables 1-3; see papers by Miller et al., 1994a, 1994b; Miller, et al., 1996). Our purpose was to characterize the diatoms at these sites and compare their occurrence with sediments of similar age from Leg 150 (Mountain, Miller, Blum, et al., 1994) and from the East Coast of the United States (Andrews, 1978, 1988). Ninety-seven samples were examined from Atlantic City, 103 from Cape May, and 79 from Island Beach. Sampling intervals ranged from $3 \mathrm{ft}(0.93 \mathrm{~m})$ to as much as $100 \mathrm{ft}(31.2 \mathrm{~m})$, but were generally within the range of $10 \mathrm{ft}(3.1 \mathrm{~m})$; sample size varied, but was on the order of 2-3 $\mathrm{cm}^{3}$. Diatom slides were prepared using the method described by Schrader (1974) and taxonomy followed Abbott, (1987a, 1987b), Andrews, (1978, 1988), Baldauf, (1984, 1986), and Burckle, $(1972,1978)$. Where a zonal designation was warranted, the schemes of Andrews (1988) and Burckle (1996) were used.

\section{RESULTS}

Atlantic City: The interval from 14 to $291 \mathrm{ft}(4.3-90.9 \mathrm{~m})$ is either barren of diatoms or contains few diatoms, none of which are age diagnostic. At and below a depth of $399 \mathrm{ft}(124 \mathrm{~m})$, diatoms are generally abundant. The joint occurrence of Delphineis novaecaesaraea, Delphineis penelliptica, and Denticulopsis hustedtii indicate an interval equivalent to the Delphineis novaecaesaraea and Coscinodiscus (now Thalassiosira) yabei Zones of Burckle (1996), even though the latter named taxon is not present. Further, this interval is equivalent to much of East Coast Diatom Zone (ECDZ) 6 and all of ECDZ 7 of Andrews (1988).

The top of the Coscinodiscus lewisianus Zone of Burckle (1996) occurs at a depth of $454 \mathrm{ft}(141 \mathrm{~m})$. This zone extends to at least 637 $\mathrm{ft}(199 \mathrm{~m})$, but may very well occur below that. Below $199 \mathrm{~m}$, diatom occurrence becomes sporadic, with most samples being barren. Andrews (1988), using second-order correlation, tied this last occurrence to the lower part of foraminiferal Zone N11 of Blow (1969), approximately equivalent to somewhere in Magnetochron 14 (C5AA; Berggren et al., 1985). The last occurrence of Rhaphidodiscus marylandicus at a depth of $473 \mathrm{ft}(147 \mathrm{~m})$ and the co-occurrence of Delphineis novaecaesaraea and Delphineis penelliptica suggest that the interval between 454 and $473 \mathrm{ft}$ (141 and $147 \mathrm{~m}$ ) ranges from the upper part of ECDZ 2 to the lowermost part of ECDZ 6 of Andrews (1988).

Island Beach: Diatoms are present in the Island Beach hole from the surface to a depth below $347 \mathrm{ft}(108 \mathrm{~m})$. Below this level, diatoms either are not present, are present in low numbers, or are in fragments. The topmost sample (79 ft [24 m]) contains abundant diatoms, but no age-diagnostic forms other than Actinocyclus ingens were identified.

${ }^{1}$ Miller, K.G., and Snyder, S.W. (Eds.), 1997. Proc. ODP, Sci. Results, 150X: College Station, TX (Ocean Drilling Program).

${ }^{2}$ Lamont-Doherty Earth Observatory, Palisades, NY 10964, U.S.A burckle@LDEO.Columbia.edu
This sample is tentatively placed in the upper Miocene. The samples below that level $(88 \mathrm{ft}[27 \mathrm{~m}])$ to a depth of $347 \mathrm{ft}(108 \mathrm{~m})$ generally contain abundant diatoms, including sporadic occurrences of $C$. lewisianus, D. novaecaesaraea, D. penelliptica, and Rhaphidodiscus marylandicus. Burckle (1996) reported this latter species in his C. lewisianus Zone. The interval between 88 and $347 \mathrm{ft}$ (27 and $108 \mathrm{~m})$ is placed in the C. lewisianus Zone of Burckle (1996) and in the lower part of ECDZ 2 and possible ECDZ 1 of Andrews (1988).

Cape May: Diatoms are generally present in the upper part of the Cape May hole, although some samples were barren. Below a level of $750 \mathrm{ft}(234 \mathrm{~m})$, diatoms are generally not present, occurring in only a few samples. Those present in samples between the top of the hole and a depth of $412 \mathrm{ft}(128 \mathrm{~m})$ are generally near-shore, brackish water forms. Below that level, a number of zonal markers appear. The interval between 412 and $658 \mathrm{ft}(128$ and $205 \mathrm{~m})$ contains D. novaecaesarae $a$ and $D$. penelliptica as well as sporadic occurrences of $D$. hustedtii. This interval is probably equivalent to the $D$. novaecaesaraea and C. yabei Zones of Burckle (1996). The absence of C. lewisianus and the presence of the aforementioned taxa suggest that it is equivalent to most of the ECDZ 6 and all of ECDZ 7 of Andrews (1988). The last occurrence of $C$. lewisianus and $R$. marylandicus at $658 \mathrm{ft}$ $(205 \mathrm{~m})$ indicates that this site penetrated into the $C$. lewisianus Zone of Burckle (1996) and ECDZ 1 and the lower half of ECDZ 2 of Andrews (1988).

\section{REFERENCES}

Abbott, W.H., 1987a. Diatom occurrences, Deep Sea Drilling Project Site 604. In van Hinte, J.E., Wise, S.W., Jr., et al. Init. Repts. DSDP, 93: Washington (U.S. Govt. Printing Office), 789-792.

1987b. Diatom occurrences, Deep Sea Drilling Project Sites 612 and 613. In Poag, C.W., Watts, A.B., et al., Init. Repts. DSDP, 95: Washington (U.S. Govt. Printing Office), 417-418.

Andrews, G.W., 1978. Marine diatom sequence in Miocene strata of the Chesapeake Bay region, Maryland. Micropaleontology, 24:371-406.

, 1988. A revised marine diatom zonation for Miocene strata of the southeastern United States. Geol. Surv. Prof. Pap. U.S., 1481:1-29.

Baldauf, J.G., 1984. Cenozoic diatom biostratigraphy and paleoceanography of the Rockall Plateau region, North Atlantic, Deep Sea Drilling Project Leg 81. In Roberts, D.G., Schnitker, D., et al., Init. Repts. DSDP, 81: Washington (U.S. Govt. Printing Office), 439-478.

, 1986. Diatom biostratigraphic and paleoceanographic interpretations for the middle to high latitude North Atlantic Ocean. In Summerhayes, C.P., and Shackleton, N.J. (Eds.), North Atlantic Paleoceanography. Geol. Soc. Spec. Publ. London, 21:243-254.

Berggren, W.A., Kent, D.V., and Van Couvering, J.A., 1985. The Neogene, Part 2. Neogene geochronology and chronostratigraphy. In Snelling N.J. (Ed.), The Chronology of the Geological Record. Geol. Soc. London Mem., 10:211-260.

Burckle, L.H., 1972. Late Cenozoic planktonic diatom zones from the eastern equatorial Pacific. In Simonsen, R. (Ed.), First Symposium on Recent and Fossil Marine Diatoms. Nova Hedwegia Beih., 39:217-250.

1978. Early Miocene to Pliocene diatom datum levels for the equatorial Pacific. Proc. Second Working Group Mtg. Biostratigraphic Datum Planes, Pacific Neogene, IGCP Proj. 114. Spec. Publ., Geol. Res. Dev. Ctr., 1:25-44.

1996. Diatom biostratigraphy, Leg 150. In Mountain, G.S., Miller, K.G., Blum, P., Poag, C.W., and Twichell, D.C. (Eds.), Proc. ODP, Sci. Results, 150: College Station, TX (Ocean Drilling Program), 17-35. 
Miller, K.G., et al., 1996. Proc. ODP, Init. Repts. 150X (Suppl.): College Station, TX (Ocean Drilling Program).

Miller, K.G., Browning, J.V., Liu, C., Sugarman, P., Kent, D.V., Van Fossen, M., Queen, D., Goss, M., Gwynn, D., Mullikin, L., Feigenson, M.D., Aubry, M.-P., Burckle, L.H., 1994a. Atlantic City site report. In Miller, K.G., et al., Proc. ODP, Init. Repts. 150X: College Station (Ocean Drilling Program), 35-55.

Miller, K.G., Sugarman, P., Van Fossen, M., Liu, C., Browning, J.V., Queen, D., Aubry, M.-P., Burckle, L.H., Goss, M., and Bukry, D., 1994b. Island Beach site report. In Miller, K.G., et al., Proc. ODP, Init. Repts., 150X: College Station (Ocean Drilling Program), 5-33.
Mountain, G.S., Miller, K.G., Blum, P., et al., 1994. Proc. ODP, Init. Repts, 150: College Station, TX, (Ocean Drilling Program).

Schrader, H.-J., 1973. Proposal for a standardized method of cleaning diatom-bearing deep-sea and land-exposed marine sediments. Nova Hedwegia Beih., 45:403-409.

\section{Date of initial receipt: 17 September 1996 \\ Date of acceptance: 20 December 1996 \\ Ms 150XSR-309}

Table 1. Atlantic City diatoms.

\begin{tabular}{|c|c|c|c|}
\hline $\begin{array}{l}\text { Depth } \\
\text { (ft) }\end{array}$ & Taxonomic comments & $\begin{array}{c}\text { Depth } \\
\text { (ft) }\end{array}$ & Taxonomic comments \\
\hline 14.0 & $\begin{array}{l}\text { Cocconeis spp. } \\
\text { Diploneis bombus } \\
\text { Nitzschia granulata } \\
\text { Thalassiosira oestrupii }\end{array}$ & 469.5 & \multirow{4}{*}{$\begin{array}{l}\text { Actinocyclus ingens } \\
\text { Actinocyclus tenellus } \\
\text { Actinoptychus senarius } \\
\text { Cymatosira belgica } \\
\text { Delphineis novaecaesaraea } \\
\text { Delphineis penelliptica } \\
\text { Melosira westii } \\
\text { Paralia sulcata }\end{array}$} \\
\hline 19.5 & Nitzschia granulata & & \\
\hline 28.6 & Cocconeis spp. & & \\
\hline 42.9 & Nitzschia granulata & \multirow{4}{*}{473.5} & \\
\hline 48.9 & Cocconeis sp. & & \multirow{3}{*}{$\begin{array}{l}\text { Actinoptychus senarius } \\
\text { Delphineis novaecaesaraea } \\
\text { Delphineis penelliptica } \\
\text { Melosira westii } \\
\text { Paralia coronata } \\
\text { Paralia sulcata } \\
\text { Rhaphidodiscus marylandicus }\end{array}$} \\
\hline 67 to 291.1 & No or few diatoms & & \\
\hline 399 & $\begin{array}{l}\text { Actinocyclus ingens? } \\
\text { Actinocyclus tenellus } \\
\text { Actinoptychus senarius }\end{array}$ & & \\
\hline \multirow{3}{*}{406.1} & $\begin{array}{l}\text { Craspedodiscus coscinodiscus } \\
\text { Delphineis novaecaesaraea } \\
\text { Melosira westii } \\
\text { Paralia sulcata } \\
\text { Xanthiopyxis oblonga }\end{array}$ & 481.7 & $\begin{array}{l}\text { Actinoptychus senarius } \\
\text { Delphineis novaecaesaraea } \\
\text { Delphineis penelliptica } \\
\text { Paralia coronata } \\
\text { Paralia sulcata }\end{array}$ \\
\hline & \multirow{2}{*}{$\begin{array}{l}\text { Actinoptychus senarius } \\
\text { Craspedodiscus coscinodiscus } \\
\text { Delphineis novaecaesaraea } \\
\text { Delphineis penelliptica } \\
\text { Melosira westii } \\
\text { Paralia coronata } \\
\text { Thalassiosira lineata } \\
\text { Xanthiopyxis oblonga }\end{array}$} & 489.8 & $\begin{array}{l}\text { Actinoptychus senarius } \\
\text { Delphineis penelliptica }\end{array}$ \\
\hline & & 495.9 & $\begin{array}{l}\text { Actinoptychus senarius } \\
\text { Coscinodiscus marginatus } \\
\text { Melosira westii } \\
\text { Cavitatus jouseana }\end{array}$ \\
\hline 413.6 & $\begin{array}{l}\text { Delphineis novaecaesaraea } \\
\text { Delphineis penelliptica } \\
\text { Melosira westii } \\
\text { Paralia coronata }\end{array}$ & 503.9 & $\begin{array}{l}\text { Actinocyclus ingens } \\
\text { Actinoptychus senarius } \\
\text { Denticulopsis norwegica } \\
\text { Diploneis bombus } \\
\text { Paralia sulcata }\end{array}$ \\
\hline \multirow[t]{2}{*}{421.9} & $\begin{array}{l}\text { Cymatosira belgica }(?) \\
\text { Delphineis novaecaesaraea } \\
\text { Delphineis penelliptica } \\
\text { Denticulopsis hustedtii } \\
\text { Melosira westii } \\
\text { Paralia coronata }\end{array}$ & 509.6 & $\begin{array}{l}\text { Actinocyclus tenella } \\
\text { Coscinodiscus lewisianus } \\
\text { Melosira westii } \\
\text { Paralia sulcata } \\
\text { Rhizosolenia barboi }\end{array}$ \\
\hline & $\begin{array}{l}\text { Triceratium subrotundum } \\
\text { Delphineis penelliptica }\end{array}$ & 521.1 & $\begin{array}{l}\text { Paralia coronata } \\
\text { Paralia sulcata }\end{array}$ \\
\hline 421.1 & Few diatoms & \multirow[t]{2}{*}{526} & \multirow{2}{*}{$\begin{array}{l}\text { Actinocyclus tenella } \\
\text { Actinoptychus senarius } \\
\text { Delphineis penelliptica? } \\
\text { Melosira westii } \\
\text { Paralia coronata } \\
\text { Paralia sulcata } \\
\text { Synedra caduceus }\end{array}$} \\
\hline 433 & $\begin{array}{l}\text { Delphineis novaecaesaraea } \\
\text { Delphineis peneliptica } \\
\text { Melosira westii } \\
\text { Paralia coronata } \\
\text { Paralia sulcata } \\
\text { Thalassiosira lineata }\end{array}$ & & \\
\hline 441.6 & $\begin{array}{l}\text { Few diatom fragments } \\
\text { Sponge spicules }\end{array}$ & 539 & $\begin{array}{l}\text { Actinocyclus tenella } \\
\text { Melosira westii } \\
\text { Xanthiopyxis oblonga }\end{array}$ \\
\hline 448.4 & $\begin{array}{l}\text { Delphineis novaecaesaraea } \\
\text { Few diatoms }\end{array}$ & \multirow[t]{2}{*}{551.1} & \multirow{2}{*}{$\begin{array}{l}\text { Actinocyclus ingens } \\
\text { Actinoptychus senarius } \\
\text { Delphineis penelliptica } \\
\text { Diploneis bombus } \\
\text { Melosira westii } \\
\text { Paralia coronata }\end{array}$} \\
\hline 454.1 & $\begin{array}{l}\text { Coscinodiscus lewisianus } \\
\text { Rhizosolenia barboi } \\
\text { few broken diatoms }\end{array}$ & & \\
\hline 457.8 & $\begin{array}{l}\text { Actinocyclus ingens } \\
\text { Actinoptychus senarius } \\
\text { Coscinodiscus lewisianus } \\
\text { Delphineis novaecaesaraea } \\
\text { Delphineis penelliptica } \\
\text { Melosira westii } \\
\text { Paralia coronata } \\
\text { Xanthiopyxis oblonga }\end{array}$ & 564.1 & $\begin{array}{l}\text { Delphineis penelliptica } \\
\text { Melosira westii } \\
\text { Paralia coronata } \\
\text { Synedra caduceus }(?)\end{array}$ \\
\hline
\end{tabular}

\begin{tabular}{|c|c|}
\hline $\begin{array}{l}\text { Depth } \\
\text { (ft) }\end{array}$ & Taxonomic comments \\
\hline 569.7 & $\begin{array}{l}\text { Actinoptychus senarius } \\
\text { Melosira westii } \\
\text { Paralia coronata } \\
\text { Paralia sulcata }\end{array}$ \\
\hline 575.8 & $\begin{array}{l}\text { Melosira westii } \\
\text { Paralia sulcata }\end{array}$ \\
\hline 583.7 & $\begin{array}{l}\text { Actinocyclus ingens } \\
\text { Actinoptychus senarius } \\
\text { Delphineis penelliptica } \\
\text { Paralia coronata } \\
\text { Paralia sulcata }\end{array}$ \\
\hline 591.4 & $\begin{array}{l}\text { Diploneis bombus } \\
\text { Paralia coronata } \\
\text { Paralia sulcata }\end{array}$ \\
\hline 607.8 & $\begin{array}{l}\text { Melosira westii } \\
\text { Paralia coronata } \\
\text { Paralia sulcata }\end{array}$ \\
\hline 619.8 & $\begin{array}{l}\text { Actinoptychus senarius } \\
\text { Melosira westii } \\
\text { Paralia sulcata } \\
\text { Xanthiopyxis oblonga }\end{array}$ \\
\hline 629.9 & $\begin{array}{l}\text { Actinocyclus ehrenbergii } \\
\text { Melosira westii } \\
\text { Paralia coronata } \\
\text { Paralia sulcata }\end{array}$ \\
\hline 637.4 & $\begin{array}{l}\text { Actinocyclus tenella } \\
\text { Melosira westii } \\
\text { Paralia coronata } \\
\text { Paralia sulcata }\end{array}$ \\
\hline 644.1 & Few diatom fragments \\
\hline 650.9 & Melosira westii \\
\hline 659.6 & $\begin{array}{l}\text { Actinocyclus ingens } \\
\text { Actinocyclus tenellus } \\
\text { Delphineis penelliptica } \\
\text { Melosira westii } \\
\text { Paralia coronata }\end{array}$ \\
\hline 663.8 to 750 & No diatoms \\
\hline 755.9 & $\begin{array}{l}\text { Actinoptychus senarius } \\
\text { Delphineis novaecaesaraea } \\
\text { Denticulopsis hustedtii } \\
\text { Diploneis bombus }\end{array}$ \\
\hline 768.7 & $\begin{array}{l}\text { Actinoptychus senarius } \\
\text { Melosira westii } \\
\text { Paralia coronata } \\
\text { Paralia sulcata }\end{array}$ \\
\hline 776 to 977 & No diatoms or few diatoms \\
\hline
\end{tabular}


Table 2. Island Beach diatoms, Hole 1B.

\begin{tabular}{|c|c|c|c|c|c|}
\hline $\begin{array}{l}\text { Depth } \\
\text { (ft) }\end{array}$ & Taxonomic comments & $\begin{array}{l}\text { Depth } \\
\text { (ft) }\end{array}$ & Taxonomic comments & $\begin{array}{l}\text { Depth } \\
(\mathrm{ft})\end{array}$ & Taxonomic comments \\
\hline \multirow[t]{2}{*}{79.8 to 79.9} & $\begin{array}{l}\text { Actinocyclus tenellus } \\
\text { Actinocyclus ingens } \\
\text { Actinoptychus senarius } \\
\text { Coscinodiscus radiatus } \\
\text { Coscinodiscus oculus-iridus } \\
\text { Craspedodiscus sp. } \\
\text { Delphineis biseriata }\end{array}$ & 111.2 to 111.3 & $\begin{array}{l}\text { Actinoptychus senarius } \\
\text { Cymatogonia amblyoceras } \\
\text { Diploneis bombus } \\
\text { Paralia sulcata } \\
\text { Stephanopyxis turris } \\
\text { Thalassionema nitzschioides } \\
\text { Thalassionema obtusa }\end{array}$ & 143.5 to 143.6 & $\begin{array}{l}\text { Actinocylcus octonarius } \\
\text { Asteromphalus imbricatus } \\
\text { Aulacodiscus sp. } \\
\text { Aulocoseira italica } \\
\text { Coscinodiscus asteromphalus } \\
\text { Coscinodiscus cf. C. lewisianus } \\
\text { Coscinodiscus lewisianius }\end{array}$ \\
\hline & $\begin{array}{l}\text { Delphineis ovata } \\
\text { Delphineis surirella } \\
\text { Diploneis bombus } \\
\text { Melosira westii } \\
\text { Paralia sulcata } \\
\text { Thalassionema obtusa }\end{array}$ & 115.2 to 115.3 & $\begin{array}{l}\text { Actinocyclus ingens } \\
\text { Actinoptychus senarius } \\
\text { Paralia sulcata } \\
\text { Rhaphoneis scalaris } \\
\text { Sceptroneis grandis } \\
\text { Stephanopyxis sp. }\end{array}$ & & $\begin{array}{l}\text { Coscinodiscus perforatus } \\
\text { Cymatosira belgica } \\
\text { Diploneis bombus } \\
\text { Melosira westii } \\
\text { Paralia complexa } \\
\text { Paralia coronata }\end{array}$ \\
\hline \multirow[t]{4}{*}{88} & $\begin{array}{l}\text { Actinocyclus tenellus } \\
\text { Actinocyclus ingens }\end{array}$ & & $\begin{array}{l}\text { Thalassionema nitzschioides } \\
\text { Thalassionema obtusa }\end{array}$ & & $\begin{array}{l}\text { Pleurosigma manni } \\
\text { Pyxilla johnsoniana } \\
\text { Rhaphoneis gemmifera }\end{array}$ \\
\hline & $\begin{array}{l}\text { Actinocyclus octonarius } \\
\text { Actinoptychus senarius } \\
\text { Azpeitia nodulifera } \\
\text { Coscinodiscus radiatus } \\
\text { Coscinodiscus oculus-iridus }\end{array}$ & 119.5 to 119.6 & $\begin{array}{l}\text { Actinoptychus senarius } \\
\text { Coscinodiscus perforatus } \\
\text { Diploneis bombus } \\
\text { Epithemia } \mathrm{sp} .\end{array}$ & & $\begin{array}{l}\text { Rhaphoneis scalaris } \\
\text { Rhizosolenia barboi } \\
\text { Stephanopyxis turris } \\
\text { Thalassiosira eccentrica }\end{array}$ \\
\hline & $\begin{array}{l}\text { Craspedodiscus } \mathrm{sp} . \\
\text { Delphineis biseriata } \\
\text { Delphineis ovata } \\
\text { Delphineis penelliptica } \\
\text { Delphineis surirella }\end{array}$ & & $\begin{array}{l}\text { Paralia sulcata } \\
\text { Podosira stelligera } \\
\text { Rhaphoneis scalaris } \\
\text { Thalassionema nitzschioides } \\
\text { Thalassionema obtusa }\end{array}$ & 144.0 to 144.1 & $\begin{array}{l}\text { Actinoptychus marylandicus } \\
\text { Actinoptychus senarius } \\
\text { Cavitatus jouseana } \\
\text { Coscinodiscus lewisianus } \\
\text { Coscinodiscus perforatus }\end{array}$ \\
\hline & $\begin{array}{l}\text { Diploneis bombus } \\
\text { Melosira westii } \\
\text { Paralia sulcata } \\
\text { Pleurosigna affine var. marylandica } \\
\text { Rhaphidodiscus marylandicus } \\
\text { Rhizosolenia styliformis } \\
\text { Stephanopyxis turris } \\
\text { Thalassionema obtusa } \\
\text { Triceratium condecorum }\end{array}$ & 123.6 to 123.7 & $\begin{array}{l}\text { Actinocyclus ingens } \\
\text { Diploneis bombus } \\
\text { Mediaria splendida } \\
\text { Paralia sulcata } \\
\text { Rhaphoneis amphiceras } \\
\text { Rhaphoneis scalaris } \\
\text { Rhyzosolenia styliformis } \\
\text { Thalassionema nitzschioides } \\
\text { Thalassionema obtusa }\end{array}$ & & $\begin{array}{l}\text { Cymatosira immunis } \\
\text { Delphineis penelliptica } \\
\text { Denticulopsis norwegica } \\
\text { Diploneis bombus } \\
\text { Paralia sulcata } \\
\text { Rhaphoneis scalaris } \\
\text { Sceptroneis grandis } \\
\text { Stephanopyxis turris } \\
\text { Thalassionem nitzschioides }\end{array}$ \\
\hline 91.8 to 91.9 & $\begin{array}{l}\text { Actinoptychus senarius } \\
\text { Coscinodiscus oculus-iridus } \\
\text { Diploneis bombus } \\
\text { Paralia sulcata } \\
\text { Pleurosigna sp. } \\
\text { Thalassionema obtusa }\end{array}$ & 127.9 to 128 & $\begin{array}{l}\text { Actinocylcus ingens } \\
\text { Actinocyclus tenellus } \\
\text { Actinocyclus senarius } \\
\text { Asterolampra marylandica } \\
\text { Coscinodiscus perforatus } \\
\text { Delphineis penelliptica }\end{array}$ & \multirow[t]{4}{*}{153.4} & $\begin{array}{l}\text { Thalassionema obtusa } \\
\text { Actinocyclus ingens } \\
\text { Actinocyclus octonarius } \\
\text { Actinoptychus senarius } \\
\text { Aulocoseira granulata } \\
\text { Aulocoseira italica }\end{array}$ \\
\hline 95.0 to 95.1 & No diatoms & & $\begin{array}{l}\text { Diploneis bombus } \\
\text { Epithemia } \mathrm{sp} .\end{array}$ & & $\begin{array}{l}\text { Cavitatus jouseana } \\
\text { Coscinodiscus endoi }\end{array}$ \\
\hline \multirow[t]{3}{*}{99.1 to 99.2} & \multirow{3}{*}{$\begin{array}{l}\text { Actinocyclus ingens } \\
\text { Actinoptychus senarius } \\
\text { Amphiceras sp. } \\
\text { Anaulus sp. } \\
\text { Azpeitia nodulifera } \\
\text { Coscinodiscus marginatus } \\
\text { Delphineis penelliptica } \\
\text { Diploneis bombus } \\
\text { Paralia sulcata } \\
\text { Rhaphidodiscus marylandicus } \\
\text { Rhaphoneis gemmifera } \\
\text { Rhaphoneis cf. R. amphiceras } \\
\text { Rhaphoneis amphiceras } \\
\text { Sceptroneis grandis } \\
\text { Thalassionema nitzschioides } \\
\text { Thalassionema obtusa }\end{array}$} & & $\begin{array}{l}\text { Lithodesmium cf. L. undulatum } \\
\text { Rhaphidodiscus marylandicus } \\
\text { Thalassionema nitzschioides } \\
\text { Thalassionema obtusa }\end{array}$ & & $\begin{array}{l}\text { Coscinodiscus marginatus } \\
\text { Craspedodiscus coscinodiscus } \\
\text { Cymatosira belgica } \\
\text { Delphineis ovata }\end{array}$ \\
\hline & & 132 to 132.1 & $\begin{array}{l}\text { Asterolampra marylandica } \\
\text { Coscinodiscus asteromphalus } \\
\text { Coscinodiscus perforatus } \\
\text { Delphineis penelliptica } \\
\text { Denticula norwegica } \\
\text { Diploneis bombus } \\
\text { Paralia sulcata } \\
\text { Rhaphoneis scalaris } \\
\text { Rhaphoneis amphiceras }\end{array}$ & & $\begin{array}{l}\text { Diploneis bombus } \\
\text { Melosira westii } \\
\text { Paralia coronata } \\
\text { Pyxilla johnsoniana } \\
\text { Rhaphidodiscus } \text { cf. } R \text {. marylandicus } \\
\text { Rhaphoneis diamontella } \\
\text { Rhaphoneis rhombica } \\
\text { Rhizosolenia barboi } \\
\text { Stephanopyxis turris }\end{array}$ \\
\hline & & & $\begin{array}{l}\text { Rhaphonets amphiceras } \\
\text { Sceptroneis sp. } \\
\text { Sceptroneis grandis } \\
\text { Thalassionema nitzschioides }\end{array}$ & 161.5 to 161.6 & $\begin{array}{l}\text { Melosira westii } \\
\text { Podosira stelligera } \\
\text { Xanthiopyxis oblonga }\end{array}$ \\
\hline \multirow[t]{2}{*}{103.2 to 103.3} & \multirow[b]{2}{*}{$\begin{array}{l}\text { Actincyclus ingens } \\
\text { Actinoptychus senarius } \\
\text { Coscinodiscus argus } \\
\text { Diploneis bombus } \\
\text { Delphineis cf. D. surirella } \\
\text { Paralia sulcata } \\
\text { Rhaphoneis amphiceras } \\
\text { Rhaphoneis scalaris } \\
\text { Stephanopyxis turris } \\
\text { Thalassionema nitzschioides } \\
\text { Thalassionema obtusa }\end{array}$} & & Thalassionema obtusa & & $\begin{array}{l}\text { Coscinodiscus perforatus } \\
\text { Triceratium condecorum }\end{array}$ \\
\hline & & 136.3 to 136.4 & $\begin{array}{l}\text { Actinoptychus senarius } \\
\text { Biddulphia } \text { cf. B. aurita } \\
\text { Coscinodiscus lewisianus } \\
\text { Coscinodiscus perforatus } \\
\text { Delphineis penelliptica } \\
\text { Denticulopsis norwegica } \\
\text { Diploneis bombus } \\
\text { Rhizosolenia miocenica } \\
\text { Rhyzosolenia styliformia } \\
\text { Thalassionema nitzschioides }\end{array}$ & 168.6 to 168.7 & $\begin{array}{l}\text { Actinocyclus ehrenbergii } \\
\text { Aulacodiscus } \mathrm{sp} \text {. } \\
\text { Coscinodiscus lewisianus } \\
\text { Coscinodiscus } \mathrm{cf} \text {. C. perforatus } \\
\text { Delphineis } \mathrm{cf} \text {. D. ovata } \\
\text { Melosira westii } \\
\text { Paralia sulcata } \\
\text { Xanthiopyxis oblonga } \\
\text { Xanthiopyxis ovalis }\end{array}$ \\
\hline \multirow[t]{2}{*}{107.5 to 107.6} & \multirow[b]{2}{*}{$\begin{array}{l}\text { Actinoptychus australis } \\
\text { Actinoptychus senarius } \\
\text { Coscinodiscus marginatus } \\
\text { Coscinodiscus radiatus } \\
\text { Diploneis bombus } \\
\text { Paralia sulcata } \\
\text { Rhaphoneis gemmifera } \\
\text { Sceptroneis grandis } \\
\text { Stephanopyxis sp. } \\
\text { Thalassionema nitzschioides } \\
\text { Thalassionema obtusa }\end{array}$} & & Thalassionema obtusa & & Bolboforma sp. (not a diatom) \\
\hline & & 140.2 to 140.3 & $\begin{array}{l}\text { Actinocyclus tenellus } \\
\text { Actinoptychus senarius } \\
\text { Asterolampra marylandica } \\
\text { Delphineis penelliptica } \\
\text { Denticulopsis norwegica } \\
\text { Epithemia sp. } \\
\text { Rhaphoneis gemmifera } \\
\text { Rhaphoneis amphiceras } \\
\text { Rhizosolenia cf. R. miocenica } \\
\text { Thalassionema nitzschioides } \\
\text { Thalassionema obtusa }\end{array}$ & $\begin{array}{l}175.6 \text { to } 175.7 \\
\text { and } \\
181.5 \text { to } 181.6\end{array}$ & Diatom fragments or no diatoms \\
\hline
\end{tabular}


Table 2 (continued).

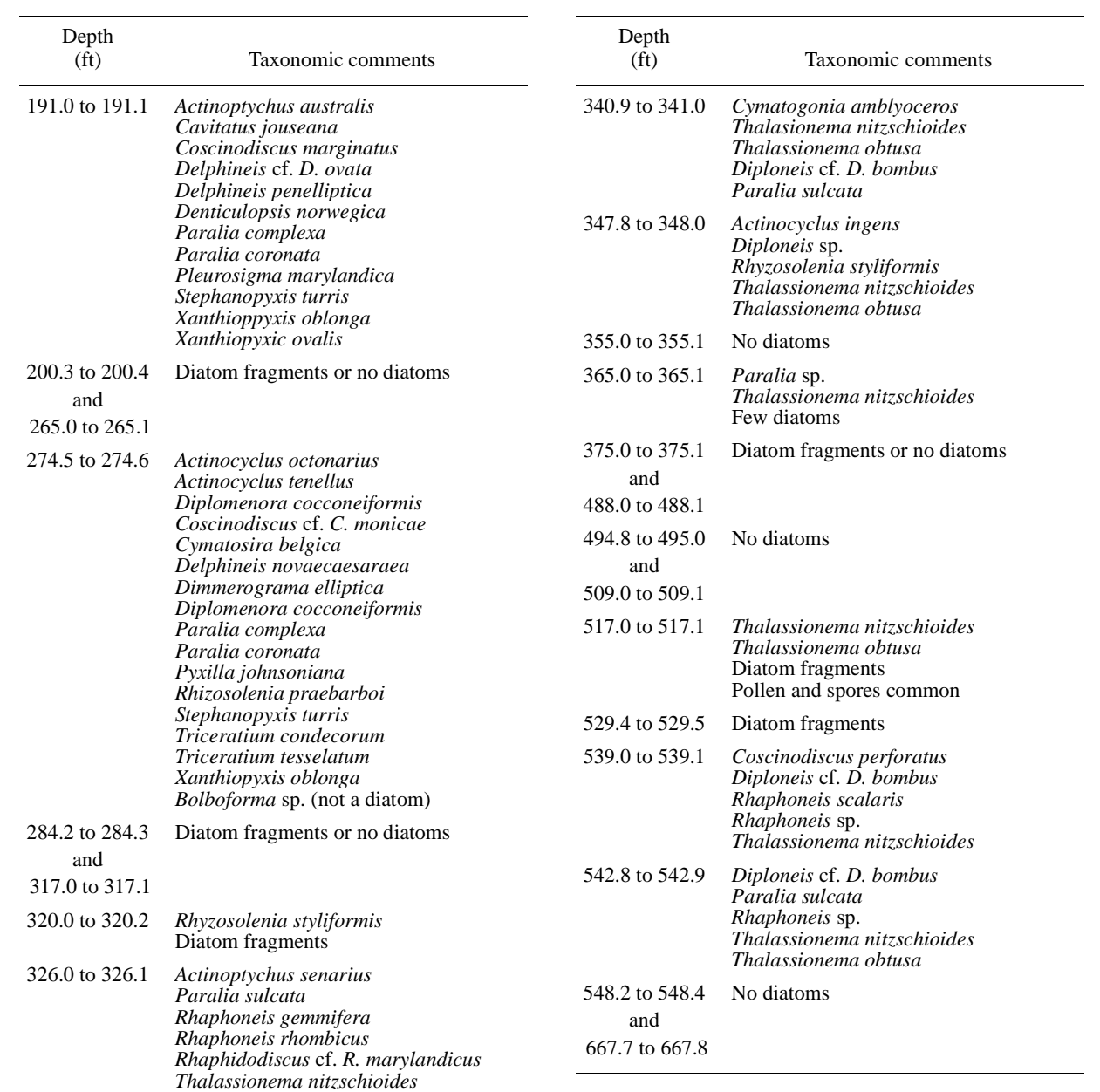

333.0 to 333.2 Actinocyclus tenellus

Actinoptychus senarius Cocconeis sp.

Coscinodiscus lewisianus

Coscinodiscus perforatus

Delphineis penelliptica

Diploneis bombus

Epithemia sp.

Paralia sulcata

Rhaphoneis scalari

Sceptroneis grandis

Thalassionema nitzschioides

Thalassionema obtusa

Thalassiosira sp. 
Table 3. Cape May diatoms.

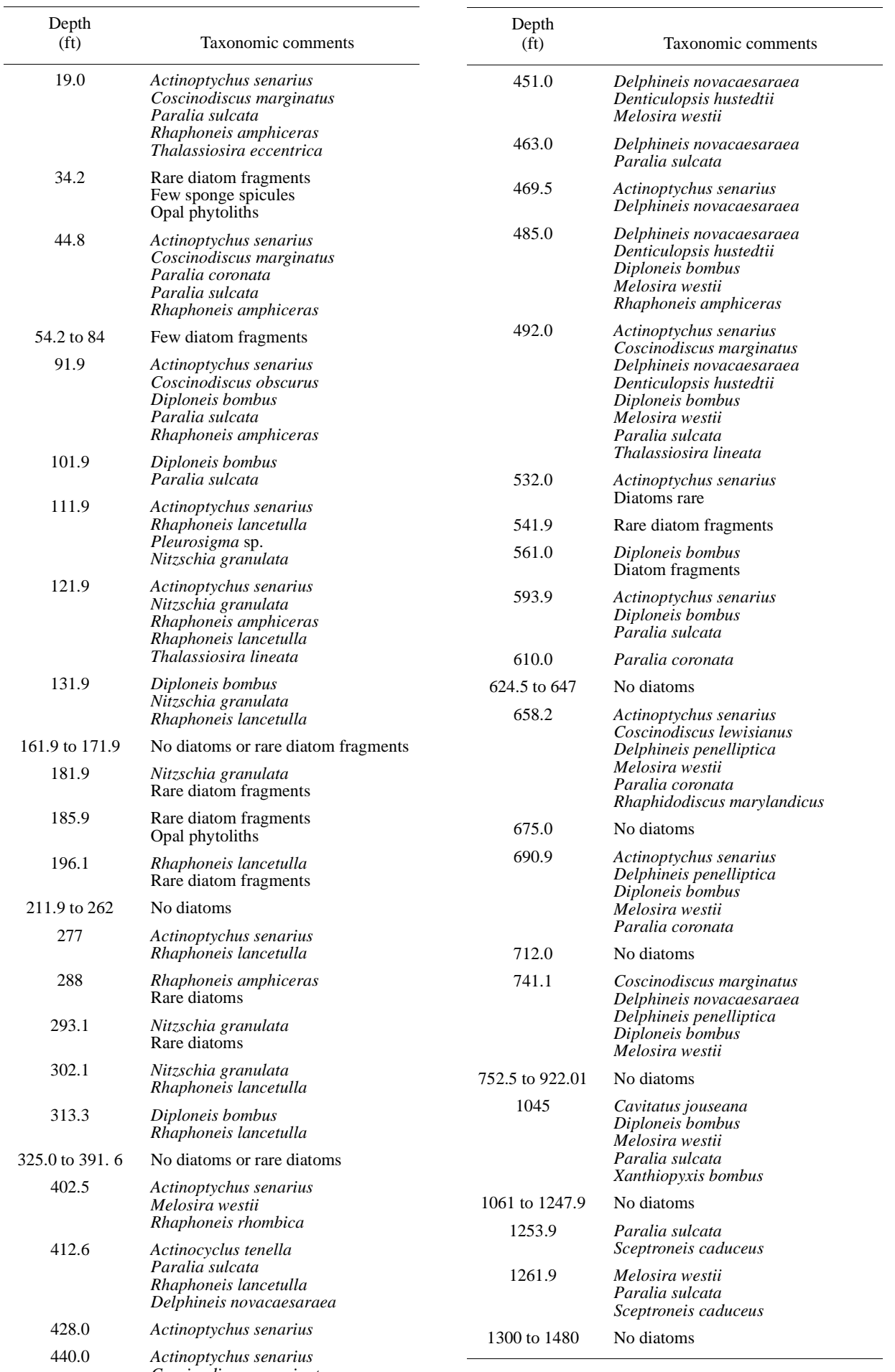

